\title{
Respuesta a la carta al editor en relación al artículo: "Estrategia de uno en uno para mejorar la técnica correcta de higiene de manos"
}

\author{
Response to the letter to the editor in \\ relation to the article: "One-on-one strat- \\ egy to improve the correct hand hygiene \\ technique".
}

Estimado editor:

En respuesta a la carta enviada por Antonella Carpio-Rodríguez y Sofía Mercado-Gonzáles del trabajo: "Estrategia de uno en uno para mejorar la técnica correcta de higiene de manos", publicado en el volumen 38, numero 5 , le comentamos que el personal de la unidad de vigilancia epidemiológica se refiere a una enfermera del Comité, por lo cual no se requirió realizar concordancia y calibración del evaluador; sin embargo, su segundo comentario sobre el sesgo del observador no se controló. En el estudio se describe que el personal evaluador acude al servicio y observa la técnica en el personal en ese momento.

La observación que se hace de utilizar una cámara seria excelente para tener una evidencia visual de la realización de higiene de manos, pero exactamente este estudio se realiza, porque los países latinoamericanos no tenemos los recursos para tener esa infraestructura y la finalidad es lograr el cambiar la conducta del personal concientizándolo como una medida tan simple para prevenir las infecciones asociadas a la atención de la salud.

La eliminación de participantes fue secundaria a que en el momento de iniciar la etapa de seguimiento se encontraban en periodo vacacional, por lo que no pensamos que los resultados se hayan afectado y no consideramos en ningún momento realizar un análisis de "intención de tratar" (intent to treat).

Agradecimos la atención y el interés por el escrito y los invitamos a continuar con la comunicación enviando sus comentarios que enriquecen el trabajo de investigación.

Hernández-Orozco Hilda G Maestra en Ciencias. Adscrita al Departamento de Infectología. Representante presidente de Comité de Infecciones Asociados a la Atención de la Salud

Castañeda-Narváez José L Médico Especialista C adscrito Depto. de Infectología wuzhi1916@gmail.com 